\title{
A new asteroseismic diagram of solar-like stars
}

\author{
Y. K. Tang ${ }^{1,3}$, S. L. Bi ${ }^{2}$, and N. Gai ${ }^{2}$ \\ 1 Department of Physics, Dezhou University, Dezhou 253023, PR China \\ e-mail: tyk450@163.com; bisl@bnu.edu.cn \\ 2 Department of Astronomy, Beijing Normal University, Beijing 100875, PR China \\ 3 Key Lab of Biophysics in Universities of Shandong, Dezhou 253023, PR China
}

Received 8 January 2008 / Accepted 13 November 2008

\begin{abstract}
Aims. We attempt to develop a new asteroseismic diagram and investigate its characteristics, based on the ratio " $r_{01}$ " of adjacent, average, small separations.

Methods. The ratio $r_{01}$ is defined following the asymptotic formula of stellar $p$-modes. Using the low-degree, theoretical, numerical value of adiabatic frequencies of stellar model, we compute the ratio $r_{01}$ and construct an additional asteroseismic diagram.

Results. The additional asteroseismic diagram, which is similar to the C-D diagram (i.e. the famous asteroseismic HR diagram, socalled Christensen-Dalsgaard diagram), represents a composite of the average, large separations $\langle\Delta v\rangle$ and the ratio $r_{01}$ of the adjacent, small separations. A distinct separation is identified between the effects of varying both mass and age. The diagram can therefore provide information that is complementary to that provided by a C-D diagram, which can be used to estimate directly both the stellar mass and evolutionary state. Interestingly, the age of star can be derived from the new asteroseismic diagram. Furthermore, we investigate the parameter $r_{01}$, which can be a useful direct indicator of stellar age.
\end{abstract}

Key words. stars: evolution - stars: oscillations

\section{Introduction}

Asteroseismology is a powerful method for both determining the fundamental properties and providing a means of studying the interior of stars, which can help us to understand both the stellar internal structure and stellar evolution. The success of helioseismology has enabled us to derive a wealth of information about the internal structure of the Sun. This is because the frequencies of oscillations depend on the sound speed inside the star, which in turn depends on density, temperature, gas motion, and other properties of the stellar interior. Therefore, the analysis of the oscillations provides an unrivaled method for probing the internal structure, and provides strong constraints on the fundamental parameters of a star. These results stimulated various attempts to detect solar-like oscillations for a handful of solar-like stars. However, the detection of solar-like oscillations is difficult because of their small amplitude. So far, seismic observations of distant stars, either from the ground or space, are likely to permit the detection of only low degree modes of oscillations $(l=0,1,2,3)$. Therefore, to extract the maximum amount of information contained in these modes, it is necessary to develop or adapt the existing seismic tools of analysis.

We can derive knowledge about the stellar interior from limited modes $(l=0,1,2,3)$, observable from solar-like oscillations. To be able to derive the largest amount of information from data of the observed frequencies, many authors have developed diagnostic tools for probing the stellar interior and constraining the model parameters (Christensen-Dalsgaard 1984, 1988, 1993; Ulrich 1986, 1988; Gough 1987, 1990, 2003, and many additional works), such as $\Delta v_{n, l}$ the large separation, $\delta_{2} v_{n, l}$ the second difference, $\delta v_{n, l}$ the small separation, which have the definitions:

$$
\begin{aligned}
\Delta v_{n, l} & =v_{n, l}-v_{n-1, l}, \\
\delta_{2} v_{n, l} & =v_{n+1, l}-2 v_{n, l}+v_{n-1, l}, \\
\delta v_{n, l} & =v_{n, l}-v_{n-1, l+2} .
\end{aligned}
$$

These quantities were inferred from the low-degree modes asymptotic expression. The physical interpretation of these seismic parameters can be found in Tassoul (1980) and Gough (1990). The large separation is sensitive to the characteristic frequency of the oscillation spectrum $v_{0}$, and $v_{0}$ is proportional to the characteristic frequency of a star $\omega_{g}=\sqrt{G M / R^{3}}$. Thus, in a homogeneous sequence of stars, the different characteristic frequencies of the oscillation spectrum are scaled by $\omega_{g}$, which is proportional to the mean density. Therefore, $v_{0}$ can be used with other stellar astronomical observations (such as the surface gravity: $g=G M / R^{2}$ ) to determine the radius and mass of the star (Gough 1987). The small separation depends on the variation in the sound speed in the central part of the star, and may provide information about the composition of the star in its nuclear region. Consequently, the large and small separation can be used to determine the evolutionary stage and mass of solar-like stars. Christensen-Dalsgaard $(1984,1988,1993)$ proposed the famous asteroseismic HR diagram (so-called Christensen-Dalsgaard diagram, or the C-D diagram, in short) to determine the mass and evolutionary state. To ascertain the stellar age $t$, Gough (1987) constructed a C-D diagram for evolutionary age $\tau=t / t_{\mathrm{ms}}$ by defining $t_{\mathrm{ms}}$ to be twice the time taken for the central hydrogen abundance to be reduced to half of its initial value. 
Mazumdar (2005) explored the feasibility of applying the C-D diagram to asteroseismic data and provided quantitative measures of the uncertainty associated with the results. He proposed a new type of seismic diagram, which was called the $\Delta-t$ diagram, for low- mass, main-sequence stars. The $\Delta-t$ diagram connects the acoustic locations of sharp features inside a star with the average large separations. The second difference, $\delta_{2} v_{n, l}$, is sensitive to the helium-ionization layers. By combining the information about the position of the base of the convective envelope or the HeII ionization zone with a measure of the average large separation, it is possible to constrain either the chemical composition or various parameters characterizing the physical processes in the stellar interior.

Building on the success of the C-D diagram and the $\Delta-t$ diagram, we propose to develop a new formal asteroseismic diagram, which can be an additional tool, or another function to determine the mass and the age of the low-mass main sequence stars directly. The aim of the present paper is to construct a new asteroseismic diagram that would help in determining the mass and age of solar-like stars, which is analogous to the C-D diagram. The computation method and results are described in Sect. 2. Finally, the discussion and conclusions are given in Sect. 3.

\section{Method and computations}

\subsection{The ratio $r_{01}$}

For $p$-modes in solar-like star, the usual frequency separations are the large and small separations defined by Eq. (1). To derive more information from the oscillation frequencies, Roxburgh (1993, 2003) considered the separations:

$\delta_{01}(n)=\frac{1}{8}\left(v_{n-1,0}-4 v_{n-1,1}+6 v_{n, 0}-4 v_{n, 1}+v_{n+1,0}\right)$,
$\delta_{10}(n)=-\frac{1}{8}\left(v_{n-1,1}-4 v_{n-1,0}+6 v_{n, 1}-4 v_{n+1,0}+v_{n+1,1}\right)$,

and defined the ratios $d_{i j}$ of small to large separations:

$$
\begin{array}{ll}
d_{02}(n)=\frac{\delta v_{02}(n)}{\Delta v_{1}(n)}, & d_{13}(n)=\frac{\delta v_{13}(n)}{\Delta v_{0}(n+1)}, \\
d_{01}(n)=\frac{\delta v_{01}(n)}{\Delta v_{1}(n)}, & d_{10}(n)=\frac{\delta v_{10}(n)}{\Delta v_{0}(n+1)} .
\end{array}
$$

The ratios $d_{i j}$ of small to large separations are independent of the structure of the outer layers of a star, and therefore provide a diagnostic of the stellar interior alone.

Gough (1990), Monteiro \& Thompson (1998), and Vauclair $\&$ Théado (2004) derived expressions for the second difference $\delta_{2} v_{l}(n)$ (see Eq. (1)). The second difference $\delta_{2} v_{l}(n)$ can be used to reveal the variation in the first adiabatic exponent $\gamma$, which should be dependent on the influence of the ionization of helium on the low-degree acoustic oscillation frequencies in models of solar-like stars. This quantity is a seismic diagnostic of the helium abundance of the star, and shows the clear signatures of the diffusion-induced, helium gradient.

According to the above references, we begin our investigation from a well-known asymptotic formula. The asymptotic formula for the frequency $v_{n, l}$ of a stellar $p$-mode of order $n$ and degree $l$ was given by Tassoul (1980):

$v_{n, l} \simeq\left(n+\frac{l}{2}+\epsilon\right) v_{0}-[A l(l+1)-B] v_{0}^{2} v_{n, l}^{-1}$, where, $v_{0}$ and $A$ are related to the sound speed (see Guenther \& Brown 2004), and $\epsilon$ and $B$ are constants that are functions of the equilibrium model.

Combining the definition given by Eq. (1) with the asymptotic formula in Eq. (5), both the large and small separations can be obtained. However, Soriano et al. (2007) and Soriano \& Vauclair (2008) found that the small separations in some cases became negative when they used the adiabatic code to compute the acoustic frequencies. This contradicts the so-called "asymptotic theory" developed by Tassoul (1980). To solve this problem and understand how small separations can become negative, Soriano et al. (2007) modified the approximate expression for $v_{n, l}$ :

$$
\begin{aligned}
v_{n, l} \simeq(n+ & \left.\frac{l}{2}+\frac{1}{4}+\alpha\right) \Delta v_{l}-\frac{l(l+1) \Delta v_{l}}{4 \pi^{2} v_{n, l}} \\
& \times\left[\frac{c(R)}{R}-\int_{r_{t}}^{R} \frac{1}{r} \frac{\mathrm{d} c}{\mathrm{~d} r} \mathrm{~d} r\right]-\delta \frac{\Delta v_{l}^{2}}{v_{n, l}},
\end{aligned}
$$

where, $r_{t}$ is the turning point for the $l$ mode with frequency $v_{n, l}$, $\Delta v_{l}$ is the large separation, $\alpha$ corresponds to a surface-phase shift, and $\delta$ is a function of the parameters of equilibrium. From Eq. (6), we are able to infer that the asymptotic expression of $v_{n, l}$ should be computed between the internal turning point of the waves $r_{t}$ and the stellar surface, instead of integrations from zero to $R$. This implies that modes of the different degrees $l$ can penetrate different depths into the stellar interior. Following Eq. (6), Soriano \& Vauclair (2008) derived the following approximate expressions for the $l=0--l=2$ and $l=1--l=3$ small separations:

$$
\begin{aligned}
\delta v_{02} \simeq & \left(n+\frac{1}{4}+\alpha\right)\left(\Delta v_{0}-\Delta v_{2}\right)-\frac{6 \Delta v_{2}}{4 \pi^{2} v_{n-1,2}} \int_{r_{t}}^{R} \frac{1}{r} \frac{\mathrm{d} c}{\mathrm{~d} r} \mathrm{~d} r \\
\delta v_{13} \simeq & \left(n+\frac{3}{4}+\alpha\right)\left(\Delta v_{1}-\Delta v_{3}\right)-\left(\frac{\Delta v_{1}}{2 \pi^{2} v_{n, l}}-\frac{6 \Delta v_{3}}{2 \pi^{2} v_{n-1,3}}\right) \\
& \times \int_{r_{t}}^{R} \frac{1}{r} \frac{\mathrm{d} c}{\mathrm{~d} r} \mathrm{~d} r
\end{aligned}
$$

From Eqs. (7) and (8), we are able to ascertain that the small separations are sensitive to the composition and, therefore, the structure of the core; in particular, an extreme sensitivity of the stellar core density stratification to several parameters is found. Here, we reintroduce the definition $r_{01}$, which is the ratio of the average small separation adjacent in $l$ (Tang et al. 2008):

$r_{01}=\frac{\left\langle\delta v_{0,2}\right\rangle}{\left\langle\delta v_{1,3}\right\rangle}$.

We use the frequencies corresponding to the range $n=10-30$. Using the definition given by Eq. (9), we can calculate the values of $r_{01}$. Fortunately, we find that the ratio $r_{01}$ is both tightly correlated and decreases monotonically with age (Tang et al. 2008). According to Eqs. (6)-(8) that are presented as modified by Soriano et al. (2007, 2008), we postulate that the most likely reason is that the central density increases with evolution. It can affect the modes of the lowest degrees most significantly, and particularly for modes of the lowest degrees that penetrate most deeply, and hence affect $\delta v_{0,2}$ more than $\delta v_{1,3}$, leading to the dependence of $r_{01}$ on absolute age. Finally, we find that the $r_{01}$ exhibits a monotonic dependence on absolute age, in contrast to $\delta v_{0,2}$, which can have a monotonic response to either evolutionary age or hydrogen mass fraction. 


\subsection{Computations}

The initial zero-age, main-sequence (ZAMS) models were derived from pre-main-sequence evolution models. We computed evolutionary sequences for models with masses from $0.7 M_{\odot}$ to $1.2 M_{\odot}$, extending to the end of central hydrogen burning using the Yale stellar evolution code (YREC, Guenther et al. 1992) by assuming OPAL equation-of-state tables EOS2001 (Rogers \& Nayfonov 2002), the opacities interpolated between OPAL GN93 (Iglesias \& Rogers 1996) and low temperature tables (Alexander \& Ferguson 1994), and the standard mixing-length theory for convection. Diffusion, rotation, and magnetic field were neglected in the present study since its inclusion would not alter the results of this work in a qualitative way. The subsequent pulsation analysis of low degree $p$-modes $(l=0-3)$ for selected models in each given mass was implemented using the Guenther $\&$ Demarque pulsation code with the adiabatic approximation (Guenther et al. 1992).

Based on the above method, we computed models in three cases:

- Case A: computing with initial heavy-metal abundance $Z_{i}=$ 0.02 , initial helium abundance $Y_{i}=0.28$ and mixing-length parameter $\alpha=1.7$;

- Case B: computing with initial heavy-metal abundance $Z_{i}=$ 0.02 , initial helium abundance $Y_{i}=0.28$ and mixing-length parameter $\alpha=1.5$;

- Case C: computing with initial heavy-metal abundance $Z_{i}=$ 0.02 , initial helium abundance $Y_{i}=0.25$ and mixing-length parameter $\alpha=1.7$.

In these cases, we can obtain the values of the average large separation $\langle\Delta v\rangle$ (covering the ranges of $n=10-30$ and $l=0-3$ ), the average small separation $\left\langle\delta v_{02}\right\rangle,\left\langle\delta v_{13}\right\rangle$, and the ratio $r_{01}$ defined by Eq. (9).

\subsection{Results}

Using the values obtained from the above computation, we construct three diagrams Fig. 1, Fig. 2, and Fig. 3 respectively. Figure 1 illustrates that there is a clear separation between data for models of different age and mass in case A. Each of the vertical lines in this diagram (and therefor in Figs. 2 and 3) is on evolutionary track of a given mass, which is labeled in solar units at the top of the track. Also, each of the approximately transverse lines is an isopleth of constant age, which is indicated in units of "Gyr" at the right of the line. We present this diagram as a new asteroseismic diagram for inferring directly the mass and age of a solar-like star. However, we most confront two problems. One is related to the use of the mixing-length theory. Figure 2 shows the variation in $\langle\Delta v\rangle$ and $r_{01}$ with the mixing length parameter $\alpha$. The other problem is related to the assumed initial composition of star. While the metallicity of a target star can often be measured from spectroscopic data, its helium content is unknown. Thereby, the initial helium abundance is effected by an ambiguity due to an unknown parameter concerning the chemical composition. Figure 3 shows that the different initial helium abundance $Y_{i}$ reduces the shift in the tracks. We must therefore account for having two unknowns in our models, the assumed initial abundance of helium and the mixing length parameter $\alpha$. In this work, we discuss the $\left(\langle\Delta v\rangle, r_{01}\right)$ diagram in only a qualitative way. From these diagrams, we can infer that the difference induced by the two unknowns increases with age. We understand that this will be an interesting problem in future work.

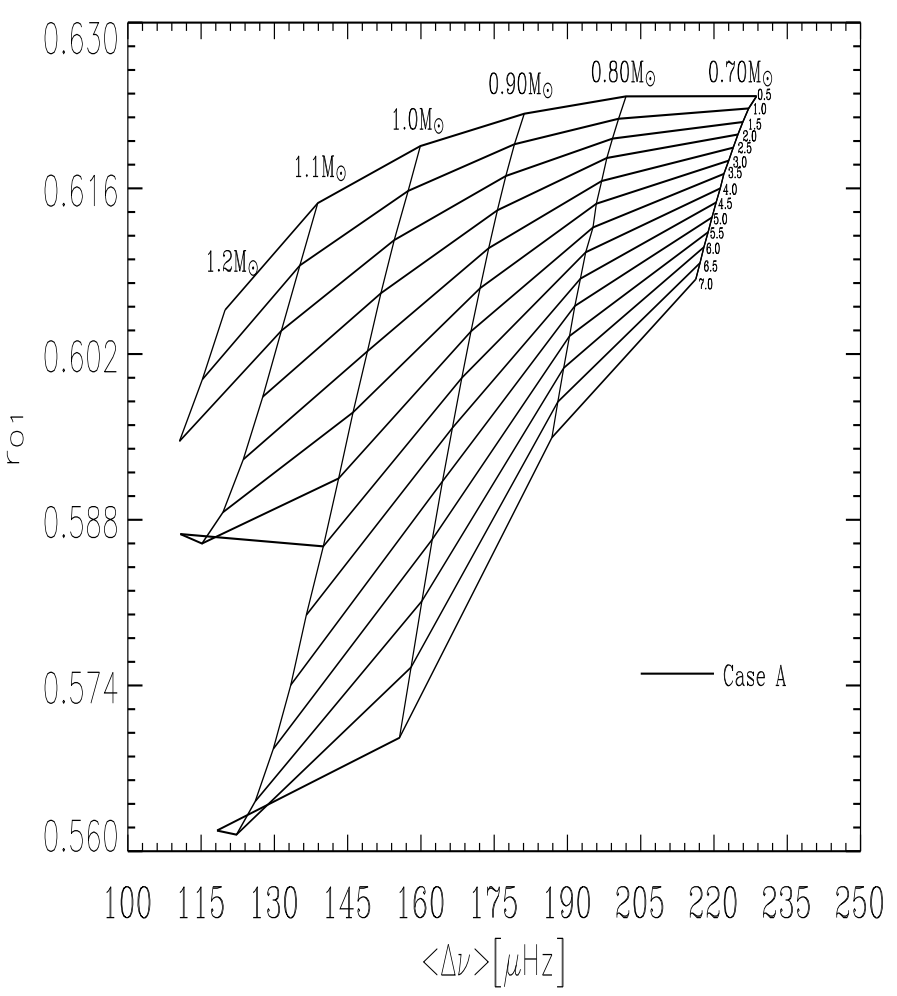

Fig. 1. $\left(\langle\Delta v\rangle, r_{01}\right)$ diagram for the models with $Y_{i}=0.28$. The vertical lines are evolutionary tracks, labeled by the mass in the top, whereas the transverse lines are isopleths with constant age, labeled by the age from $0.5 \mathrm{Gyr}$ to $7.0 \mathrm{Gyr}$, increasing in units of $0.5 \mathrm{Gyr}$.

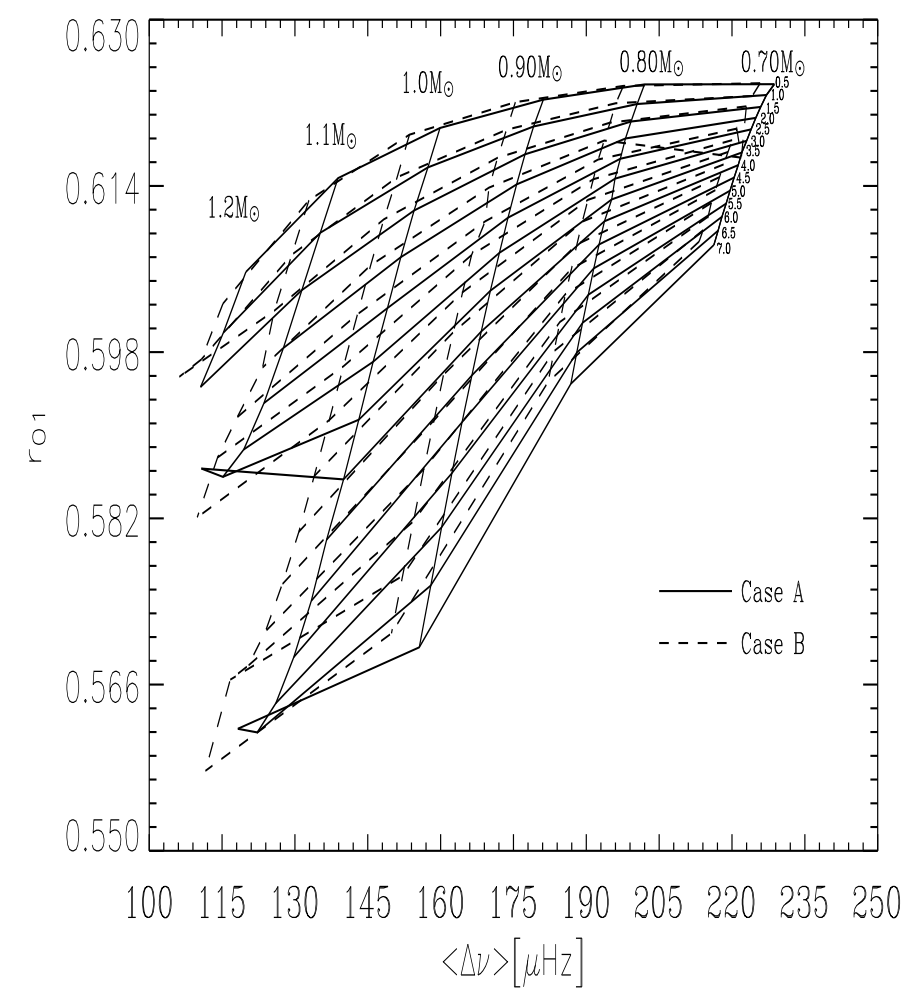

Fig. 2. $\left(\langle\Delta v\rangle, r_{01}\right)$ diagram for the models with $\alpha=1.5$ and 1.7. For comparison, the dash lines indicate the models with $\alpha=1.5$ and the solid lines $\alpha=1.7$, respectively. The vertical lines are evolutionary tracks, labeled by the mass in the top, whereas the transverse lines are isopleths with constant age, labeled by the age from $0.5 \mathrm{Gyr}$ to $7.0 \mathrm{Gyr}$, increasing in units of $0.5 \mathrm{Gyr}$. 


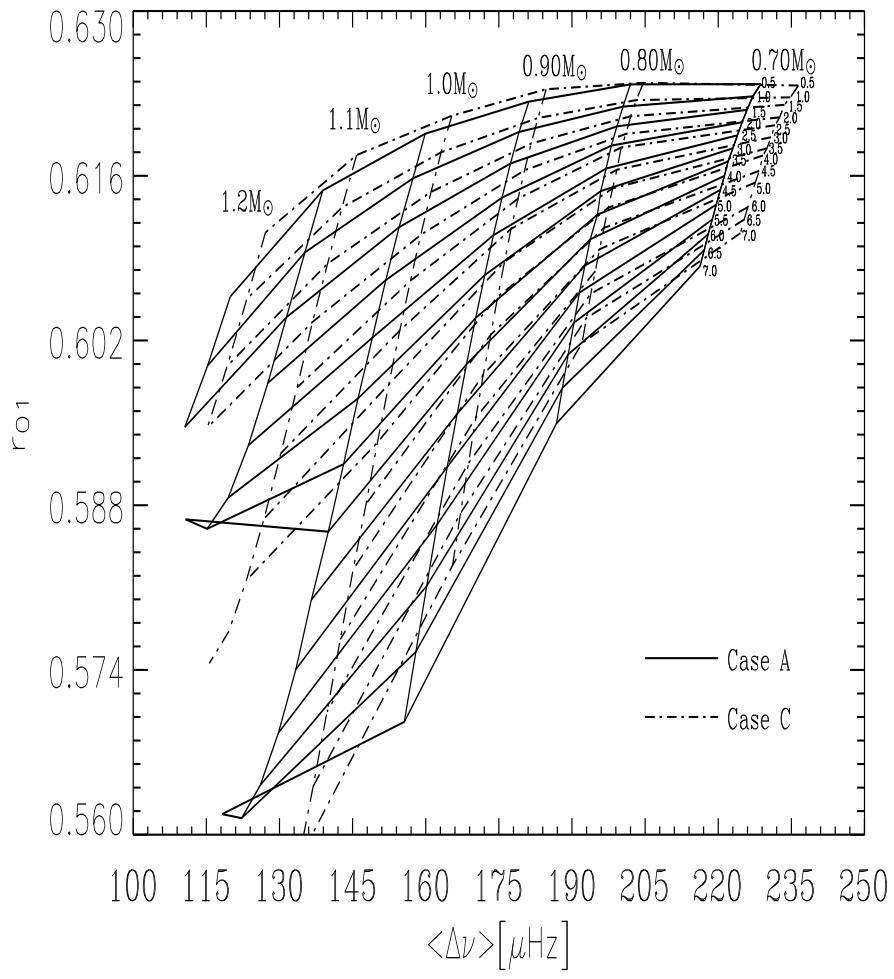

Fig. 3. $\left(\langle\Delta v\rangle, r_{01}\right)$ diagram for the models with $Y_{i}=0.25$ and 0.28 . For comparison, the dash dot lines indicate the models with $Y_{i}=0.25$ and the solid lines $Y_{i}=0.28$, respectively. The vertical lines are evolutionary tracks, labeled by the mass in the top, whereas the transverse lines are isopleths with constant age, labeled by the age from $0.5 \mathrm{Gyr}$ to $7.0 \mathrm{Gyr}$, increasing in units of $0.5 \mathrm{Gyr}$.

\section{Discussion}

Guenther (1998) highlighted that, in stellar models, we must simply accept the assumed initial abundance of helium and the mixing length parameter. Although the $\left(\langle\Delta v\rangle, r_{01}\right)$ diagram has little uncertainty in either mass or age induced by the two unknowns, it may be possible alternatively to determine the mass and age of stars from the new asteroseismic diagram. It is noticeable that the abnormal will appear as the star evolves, the mean molecular weight gradients become steeper in the core, and the frequencies of the $g$-modes increase, becoming closer to the low- $n p$-mode frequencies in these figures. The reliability of the diagram $\left(\langle\Delta v\rangle, r_{01}\right)$, as in the case of the C-D diagram and $\Delta-t$ diagram, will be reduced if the chemical composition is not fully known. Meanwhile, the structural changes in a star as it evolves off the main-sequence will affect the $p$-mode frequencies. At late stages of evolution, particularly in more massive stars, the presence of mixed non-radial modes reduce the number of modes available for asymptotic analysis. Typically, this increases the error in both the average small separations and ratio $r_{01}$, two problems that we must take into account. In other words, the $\left(\langle\Delta v\rangle, r_{01}\right)$ diagram, like the C-D diagram and the $\Delta-t$ diagram, is suitable for use with low-mass main-sequence stars.

In conclusion, the $\left(\langle\Delta v\rangle, r_{01}\right)$ diagram promises to be an important tool in interpreting present and future data sets. Many ground telescopes and the Stellar Oscillations Network Group
(SONG) will soon provide accurate seismic data on solar-like stars. Our diagram can perhaps help in estimating physical parameters of targeted stars efficiently. In addition, it may be useful in deducing the common age and composition of a cluster of stars.

Finally, our conclusions are as follows:

1. As a new asteroseismic diagnostic tool, the $\left(\langle\Delta v\rangle, r_{01}\right)$ diagram can estimate both the mass and age of solar-like stars. The virtue is that the age of stars can be marked in the diagram, so that the mass and age can be inferred directly.

2. If $\left(\langle\Delta v\rangle, r_{01}\right)$ diagram can be used in conjunction with both the C-D and $\Delta-t$ diagrams, the efficiency and precision of the measurements derived can be further enhanced, and the uncertainty in the age and mass determinations may be reduced.

The application of the additional asteroseismic $\left(\langle\Delta v\rangle, r_{01}\right)$ diagram to solar-like stars appears to be reasonable, provided that the dependence of these diagrams on different unknown stellar parameters is taken into account in the calibration process. On the other hand, the sensitivity of seismic diagrams, such the C-D diagram and $\Delta-t$ diagram, can be exploited in constructing reliable models for target stars based on model-independent results inferred from their oscillation frequencies.

Acknowledgements. We are grateful to anonymous referee for his/her constructive suggestions and valuable remarks to improve the manuscript. This work was supported by The Ministry of Science and Technology of the People's republic of China through grant 2007CB815406, and by NSFC grants 10173021, 10433030, 10773003, and 10778601 .

\section{References}

Alexander, D. R., \& Ferguson, J. W. 1994, ApJ, 437, 879

Christensen-Dalsgaard, J. 1984, In Space Research Prospects in Stellar Activity and Variability, ed. F. Praderie (Paris Observatory Press), 11

Christensen-Dalsgaard, J. 1988, in Advances in helio- and asteroseismology, ed. J. Christensen-Dalsgaard, \& S. Frandsen (Dordrecht: Reidel), Proc. IAU Symp., 123, 295

Christensen-Dalsgaard, J. 1993, in Seismic Investigation of the Sun and Stars, ed. T. M. Bron, ASP Conf. Ser., 42, 347

Gough, D. O. 1987, Nature, 326, 257

Gough, D. O. 1990, in Progress of Seismology of the Sun and Stars, Proc. Oji International Seminar Hakone (Japan: Springer Verlag), Lect. Notes Phys., 367,283

Gough, D. O. 2003, Ap\&SS, 284, 165

Guenther, D. B. 1998, in Proc. SOHO 6/GONG 98 Workshop, Structure and Dynamics of the Interior of the Sun and Sun-like Stars, ed. S. Korzennik, \& A. Wilson (ESA SP-418, Noordwijk: ESA), 375

Guenther, D. B., \& Brown, K. I. T. 2004, ApJ, 600, 419

Guenther, D. B., Demarque, P., Kim, Y.-C., et al. 1992, ApJ, 387, 372

Iglesias, C. A., \& Rogers, F. J. 1996, ApJ, 464, 943

Mazumdar, A. 2005, A\&A, 441, 1079

Monteiro, M. J. P. F. G., \& Thompson, M. J. 1998, in New Eyes to See Inside the Sun and Stars, ed. F. L. Deubner, J. Christensen-Dalsgaard, \& D. W. Kurtz (Dordrecht: Kluwer), Proc. IAU Symp., 185, 317

Rogers, F. J., \& Nayfonov, A. 2002, ApJ, 576, 1064

Roxburgh, I. W. 1993, in PRISMA, Report of Phase A Study, ed. T. Approurchaux, et al., ESA, 93, 31

Roxburgh, I. W., \& Vorontsov, S. V. 2003, A\&A, 411, 215

Soriano, M., \& Vauclair, S. 2008, A\&A, 488, 975

Soriano, M., Vauclair, S., Vauclair, G., \& Laymand, M. 2007, A\&A, 471, 885

Tang, Y. K., Bi, S. L., \& Gai, N. 2008, NewA, 13, 541

Tassoul, M. 1980, ApJS, 43, 469

Ulrich, R. K. 1986, ApJL, 306, L37

Ulrich, R. K. 1988, IAUS, 123, 299

Vauclair, S., \& Théado, S. 2004, A\&A, 425, 179 